\title{
Numerical Model of Convective Heat Transfer in a Multi-level Hydroponic Greenhouse
}

\author{
Vitaly Haslavsky ${ }^{1}$, Helena Vitoshkin² \\ ${ }^{1}$ Azrieli College of Engineering \\ 26 Yaakov Shreibom Street, Jerusalem, Israel \\ vitaliha@jce.ac.il \\ ${ }^{2}$ Agricultural Research Organization, The Volcani Center \\ P.O.Box 6, 5025001, 68 Dereh Hamacabim, Rishon lesion, Israel \\ elenav@volcani.agri.gov.il
}

\section{Extended Abstract}

Plant growth in a multi-level controlled environment allows intensive cultivation with minimal use of soil and water resources. It also reduces the risks of yield losses, as it is not affected by climate change. However, multi-level plant factories have not been widely utilized due to high energy consumption and complex design. The comprehensive review of greenhouse ventilation methods [1] pointed out the impact of vent configuration on internal climatic conditions and crop growth interactions. The importance of considering the microclimate at the leaf level as distinct from the overall indoor climate when managing the greenhouse climate for disease control was also demonstrated by [2-4]. Effective interaction between plants and the environment involves controlling temperature gradients, transpiration, carbon dioxide concentrations, and inner air velocity and velocity within the leaf boundary-layer [5]. Therefore, structural design and microclimate analysis are needed to improve air and temperature uniform distribution.

The numerical model has been developed to analyze the airflow characteristics and temperature distribution within a multi-level hydroponic grow system. The aim is to provide a uniform internal microclimate, evaluate the energy consumption of the system, and to examine options for the use of renewable energy in a controlled environment. Three-dimensional flow inside and around the multi-level unit has been modeled using ANSYS Software to explore the optimal structure elements and ventilation methods. The numerical modeling of environmental conditions between the levels includes models of turbulent forced airflow, transpiration rate, and convective heat transfer. The model of the porosity of a crop leaf layer and transpiration rates, validated by experimental measurements conducted in a commercial greenhouse. The numerical simulations describe flow regimes and temperature fields on each level. The model allows examination of the artificial lighting intensity and the effect of supplemental solar radiation on the temperature in a greenhouse.

\section{References}

[1] P.-E. Bournet, T. Boulard, Effect of ventilator configuration on the distributed climate of greenhouses: A review of experimental and CFD studies. Computers and Electronics in Agriculture, 2010, vol. 74.2, pp. 195-217.

[2] T. Boulard, H. Fatnassi, J.C. Roy, J. Lagier, J. Fargues, N. Smits, M. Rougier and B. Jeannequin, Effect of greenhouse ventilation on the humidity of inside air and in leaf boundary-layer., Agric. and For. Meteorol., 2004, vol. 125, ( Issues 3-4), pp. 225-239.

[3] T.J. Jewet, and W.R. Jarvis, Management of the greenhouse climate in relation to disease control: a review. Agronomy, 2001, vol. 21 (4), pp. 351-366.

[4] H. Vitoshkin, M. Sacks and V. Haslavsky, "Microclimate analysis to test the performance of an experimental two-level unit in the hydroponic greenhouse," in Proceedings of the ACTA III International Symposium on Soilless Culture and Hydroponics, 2020.

[5] Y.Zhang, M.Kacira, and L. An, A CFD study on improving airflow uniformity in the indoor plant factory system. Biosyst. Eng., 2016, vol. 147, pp. 193-205. 